\title{
Urachal abscess precipitated as acute pyelonephritis in an adult patient with diabetes
}

\author{
Yoo A Choi, Se Young Kim, Kyung Yoon Chang, Hyeon Seok Hwang, Suk Young Kim, and \\ Yoon Kyung Chang
}

Division of Nephrology, Department of Internal Medicine, College of Medicine, The Catholic University of Korea, Seoul, Korea

Received: December 10, 2014

Revised : February 2, 2015

Accepted: May 11, 2015

\section{Correspondence to}

Yoon Kyung Chang, M.D.

Department of Internal Medicine, College of Medicine, Daejeon St. Mary's Hospital, The Catholic University of Korea, 64 Daeheung-ro, Jung-gu, Daejeon 34943 , Korea

Tel: +82-42-220-9303

Fax: +82-42-253-9505

E-mail: racer@catholic.ac.kr
To the Editor,

The urachus or median umbilical ligament is a fibrous cord structure that forms after obliteration of the urogenital sinus and allantois during normal embryogenesis. A partial or total defect in obliteration of the urachus after 5 months of gestation can cause an urachal anomaly, which is usually discovered in childhood. Urachal diseases are rarely reported in adults, because they are difficult to find and rarely cause symptoms. Here, we present a case of a huge urachal abscess in an adult female patient with diabetes mellitus who presented with acute pyelonephritis.

A 57-year-old woman visited the emergency room for fever accompanied by pain on both flanks, lower abdominal discomfort, and dysuria aggravated by 2 days of $1-G$ cephalosporine dispensed from a local clinic to treat a urinary tract infection (UTI). She had been diagnosed with diabetes mellitus 5 years ago and was prescribed an oral hypoglycemic agent. On admission, vital signs were blood pressure 100/60 $\mathrm{mmHg}$, pulse rate 88 beats per minute, respiratory rate 20 breaths per minute, and body temperature $38.3^{\circ} \mathrm{C}$. She showed tenderness in both costovertebral angles and in the lower abdomen; however, the upper abdomen was soft and normoactive. A complete blood cell count revealed a white blood cell
(WBC) count of $13,400 / \mathrm{mm}^{3}$, a hemoglobin level of $13.3 \mathrm{~g} / \mathrm{dL}$, and a platelet count of $297,000 / \mathrm{mm}^{3}$. The glycated hemoglobin level was $8.3 \%$. The results of blood chemistry were blood urea nitrogen $11.3 \mathrm{mg} / \mathrm{dL}$, creatinine $0.46 \mathrm{mg} /$ dL, C-reactive protein $8.03 \mathrm{mg} / \mathrm{dL}$, sodium $139 \mathrm{mEq} / \mathrm{L}$, potassium $4.2 \mathrm{mEq} / \mathrm{L}$, and chloride $103 \mathrm{mEq} / \mathrm{L}$. Urine was collected with a Nelaton catheter; specific gravity was 1.030, $\mathrm{pH}$ was 5.0, protein $(-)$, red blood cells were 1 to 3 cells/high power field (HPF), and WBC were 4 to 9 cells/HPF; a few bacteria were detected. An initial simple abdominal X-ray and kidney sonography revealed normal kidney shape. Under the impression of acute pyelonephritis, she was started on intravenous ceftriaxone. Her symptoms seemed to subside gradually 3 days after admission, but fever spiked on day 4 , and she complained of severe lower abdominal pain. We immediately conducted lower abdominal sonography (Fig. 1A), which demonstrated a huge cystic abscess above the bladder. An abdominal computed tomography (CT) scan (Fig. 1B-1D) confirmed a $6 \times 5$-cmsized urachal abscess that was attached from the dome of the bladder to the umbilicus along the mid-supravesical line. It was infected and had originated from an urachal cyst. A pig-tail catheter was used to drain the abscess on day 5 using a sonographic-guided percuta- 

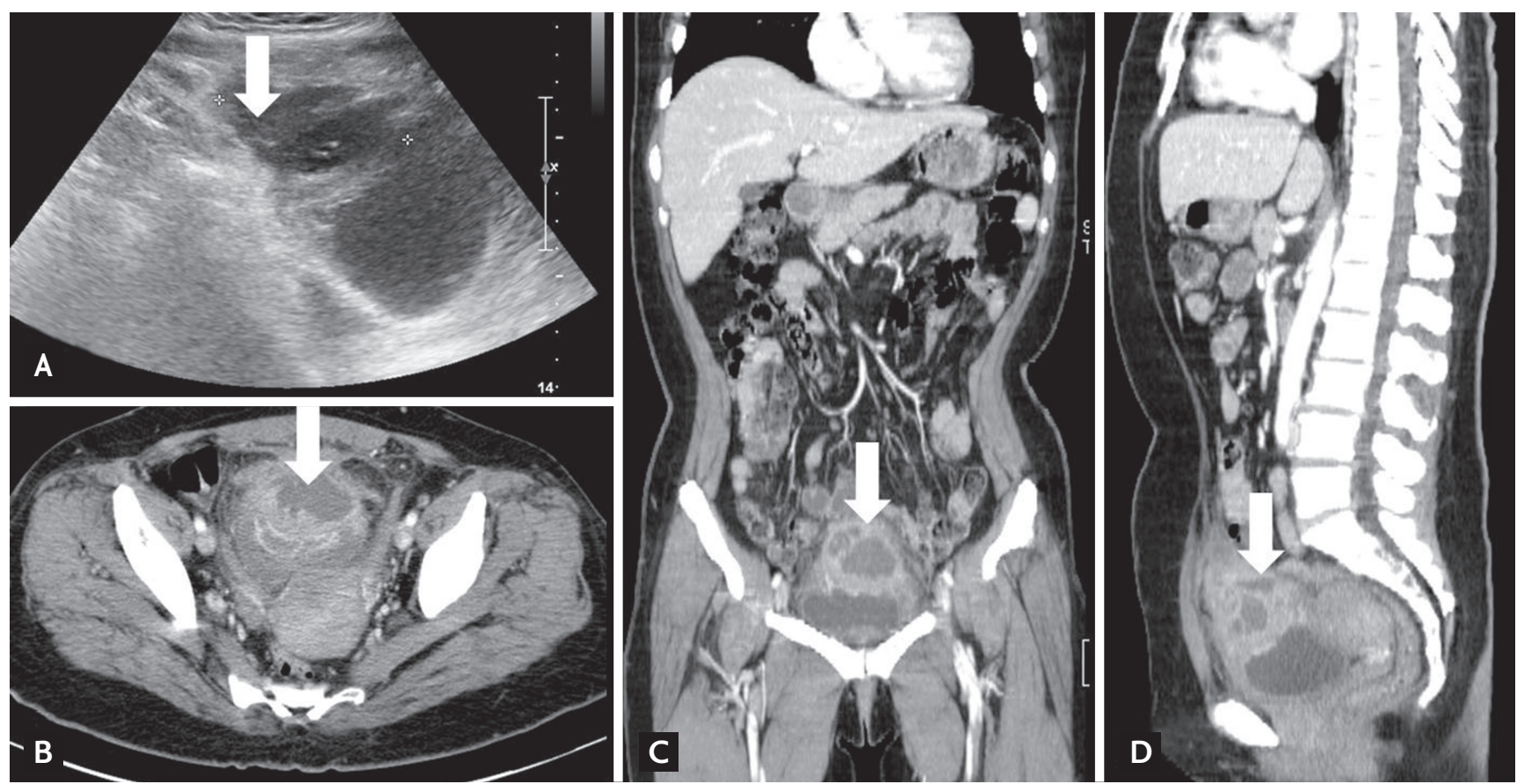

Figure 1. Abdominal images. Sonography of the lower abdomen on day 4 (A) shows a multi-septated cystic mass above the bladder. Contrast-enhanced abdominal computed tomography scan (B, transverse; C, coronal; D, sagittal) shows a $6 \times 5$-cm abscess adjacent to the urinary bladder in the mid-supravesical to umbilical area (arrows).
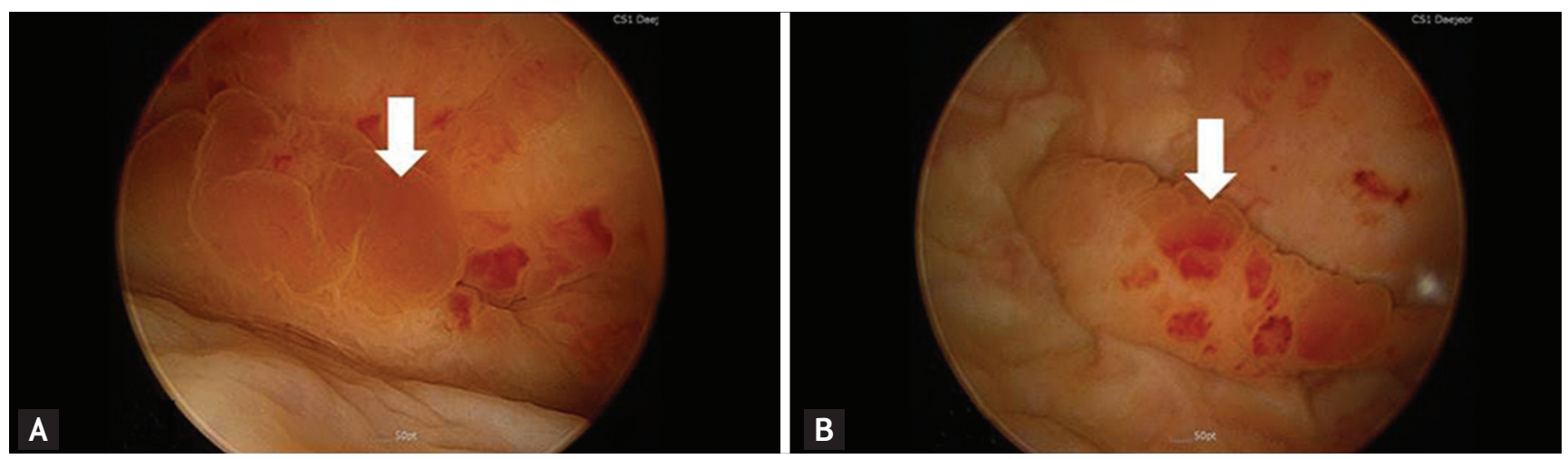

Figure 2. (A, B) Cystoscopy shows protruding mucosal masses on the anterior wall of the urinary bladder (arrows).

neous approach; $200 \mathrm{~mL}$ of pus was drained from the abscess. The blood microbiological, urine, and aspirated fluid cultures from the urachal abscess showed no microorganismal growth. Cystoscopy was conducted to rule out a combined malignancy of the abscess cavity or connection to the bladder. Some protruding masses were detected on the anterior wall of the urinary bladder (Fig. 2); however, the bladder was not connected to the abscess. The biopsy report of the bladder mass was consistent with acute and chronic inflammation, and the mass contained granulation tissue only. Urine cytology was negative for malignancy. She underwent excision surgery for the lesions under general anesthesia on hospital day 13. The urachal abscess was detached from the urinary bladder, and the bladder was well preserved. The biopsy report of the excised urachal abscess (Fig. 3) was consistent with benign features, such as acute and chronic inflammation with proliferation of granulation tissue, lipid granuloma with foamy histiocytes and fibrosis, and surrounding fibroadipose tissue with inflammatory cells. The patient was discharged on hospital day 22 without any complications. 


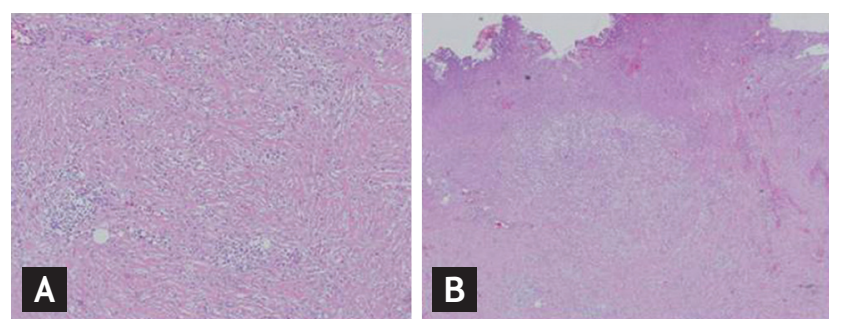

Figure 3. Histology of the excised urachal abscess. (A) The excised mass was composed of fibro-adipose tissue with inflammatory cells (H\&E, ×100). (B) The pathological findings show acute and chronic inflammation with proliferating granulation tissue $(\mathrm{H} \& \mathrm{E}, \times 4 \mathrm{O})$.

The urachus is a normal embryological tract remnant of the cloaca and allantosis and a midline fibrous cord extending from the urinary bladder to the umbilicus [1]. It occupies the potential midline space between the peritoneum and transversalis abdominis fascia. Congenital anomalies occur when the urachus fails to degenerate after birth. Four types have been classified: patent urachus (about 50\%), which connects the bladder and umbilicus; urachal cyst (about 30\%), which is a cyst-like structure within the urachus; umbilical sinus (about 15\%), in which the urachus opens into the umbilicus only; and vesicourachal diverticulum (about 5\%), in which the urachus has a wide patent opening into the bladder [2]. Congenital urachal anomalies are more common in males, at a ratio of 2:1 to 9:1 and should be suspected if umbilical discharge exists in the urine during infancy and childhood [1]. Our patient was a middle-aged woman with an infected urachal cyst that progressed to an abscess. Unfortunately, she had been treated with antibiotics blindly for 2 to 3 days, so we could not estimate the course of the progression.

Urachal diseases include urachal anomalies complicated with infections, perforations, abscesses, or malignant degeneration $[2,3]$. A patient with urachal abscess may present with umbilical discharge, fever, low abdominal pain, periumbilical redness and tenderness, hematuria, and peritonitis. The diagnosis is based on either ultrasonography or CT. Cystography or cystoscopy can be useful to define the full extent of the bladder wall and delineate the urachus [3,4]. Escherichia coli and Proteus spp. are the most common causative organisms of an urachal abscess and Staphylococcus aureus, Bacteroides spp., Fusobacterium spp., and Streptococcus viridans have also been reported. Treatment consists of antibiotics, adequate drainage, and total excision of the remnant tissue. Cellulitis, necrotizing fasciitis, peritonitis, acute appendicitis, hematoma, ventral or umbilical hernia, and tumor lesions should be considered in the differential diagnosis of an urachal abscess [4]. Interestingly, urachal anomalies can increase the risk for malignancy in adults [4]. In our case, mucosal masses were present in the anterior wall of the bladder, but were benign in nature.

UTIs are the most common and prevalent infection in women. Although diagnosing a UTI is based on urinary symptoms, urinalysis, urine culture, and imaging studies, physicians often diagnose a UTI using only symptoms or signs, and sometimes misdiagnose or fail to diagnose an unusual serious disease. A large proportion of older women are misdiagnosed with a UTI [5]. Our patient was a middle-aged woman with diabetes who initially presented with dysuria, fever, and pain on both flanks without pathognomic periumblical signs or symptoms. The initial sonography did not detect the bladder and missed the early diagnosis of an urachal abscess. She responded well to the treatment after diagnosing the urachal abscess. However, this case emphasizes the importance of recognizing rare congenital anomalies in adults and performing a bladder inspection as an integral part of an abdominal sonographic exam.

In conclusion, we emphasize that an urachal abscess may mimic a UTI without pathognomic symptoms or signs in adults. An urachal abscess should be considered in the differential diagnosis of UTI with persistent symptoms, even if appropriate antibiotic therapy has been prescribed.

Keywords: Urachal cyst; Pyelonephritis; Diabetes mellitus

\section{Conflict of interest}

No potential conflict of interest relevant to this article was reported.

\section{REFERENCES}

1. Lee SH, Lee HI, Shin DG. Urachal cyst presenting with huge abscess formation in adults. J Korean Surg Soc 2012;83:254-257.

2. Ashley RA, Inman BA, Routh JC, Rohlinger AL, Husmann DA, Kramer SA. Urachal anomalies: a longitudi- 
nal study of urachal remnants in children and adults. J Urol 2007;178(4 Pt 2):1615-1618.

3. Schnyder P, Candardjis G. Vesicourachal diverticulum: CT diagnosis in two adults. AJR Am J Roentgenol 1981; 137:1063-1065.

4. Allen JW, Song J, Velcek FT. Acute presentation of infected urachal cysts: case report and review of diagno- sis and therapeutic interventions. Pediatr Emerg Care 2004;20:108-111.

5. Gordon LB, Waxman MJ, Ragsdale L, Mermel LA. Overtreatment of presumed urinary tract infection in older women presenting to the emergency department. J Am Geriatr Soc 2013;61:788-792. 